\title{
COORDINATION of ORAL ANTICOAGULANT CARE at HOSPITAL DISCHARGE (COACHeD): Protocol for a Pilot Randomised Controlled Trial
}

Dr. Anne Holbrook ( $\sim$ holbrook@mcmaster.ca )

McMaster University

Kristina Vidug

St. Joseph's Healthcare Hamilton

Lindsay Yoo

St. Joseph's Healthcare Hamilton

Sue Troyan

St. Joseph's Healthcare Hamilton

Sam Schulman

McMaster University

James Douketis

McMaster University

Lehana Thabane

St. Joseph's Healthcare Hamilton

Stephen Giilck

McMaster University

Yousery Koubaesh

McMaster University

Sylvia Hyland

ISMP Canada

Karim Keshavjee

University of Toronto

Joanne Ho

McMaster University

Jean-Eric Tarride

McMaster University

Amna Ahmed

McMaster University

Marianne Talman

McMaster University

Blair Leonard 
Niagara Health System

Khursheed Ahmed

McMaster University

Mohammad Refaei

Niagara Health System

Deborah Siegal

University of Ottawa

\section{Research Article}

Keywords: pharmacology, oral anticoagulant, coordination of care, medication safety, randomised control trial

Posted Date: January 21st, 2022

DOI: https://doi.org/10.21203/rs.3.rs-1280258/v1

License: (c) (1) This work is licensed under a Creative Commons Attribution 4.0 International License. Read Full License 


\section{Abstract}

Background Oral anticoagulants (OACs) are commonly prescribed, have well documented benefits for important clinical outcomes but have serious harms as well. Rates of OAC-related adverse events including thromboembolic and hemorrhagic events are especially high shortly after hospital discharge. Expert OAC management involving virtual care is a research priority given its potential to reach remote communities in a more feasible, timely and less costly way than in-person care. Our objective is to test whether a focused, expert medication management intervention using a mix of in-person consultation and virtual care follow-up, is feasible and effective in preventing anticoagulation-related adverse events, for patients transitioning from hospital to home.

Methods and Analysis A randomized, parallel, multicentre design enrolling consenting adult patients or the caregivers of cognitively impaired patients about to be discharged from medical wards with a discharge prescription for an OAC. The interdisciplinary multimodal intervention is led by a clinical pharmacologist and includes: a detailed discharge medication reconciliation and management plan focused on oral anticoagulants at hospital discharge; a circle of care handover and coordination with patient, hospital team and community providers; and early post-discharge follow-up virtual medication check-up visits at 24 hours, 1 week, and 1 month. The control group will receive usual care plus encouragement to use the Thrombosis Canada website.

The primary feasibility outcomes include recruitment rate, participant retention rates, trial resources management, and the secondary clinical outcomes include adverse anticoagulant safety events composite (AASE), coordination and continuity of care, medication-related problems, quality of life and healthcare resource utilization. Follow-up is 3 months.

\section{Discussion}

This pilot RCT tests whether there is sufficient feasibility and merit in coordinating oral anticoagulant care early post-hospital discharge to warrant a full sized RCT

Trial Registration Number NCT02777047

\section{Strengths And Limitations Of This Study}

- There is a lack of high quality evidence on hospital discharge strategies which reduce oral anticoagulant (OAC) -related adverse events.

- Our methods build our prior research showing that early post-hospital discharge is a high risk period for adverse events for patients, that coordination of OAC management peri-hospital discharge is hypothesized to reduce events, and that virtual care may be more cost-effective than in person care.

- This pragmatic pilot randomized trial combines expert multidisciplinary medication management led by Clinical Pharmacology at hospital discharge with virtual care follow-up. 
- Our feasibility outcomes introduce the concept of research resource utilization and management, expressed as cost per patient completing the trial, as a key outcome.

\section{Introduction}

\section{Background and rationale}

ISMP data suggest that approximately 300,000 Canadians suffer serious, disabling or fatal medicationrelated harm annually. ${ }^{1,2}$ Anticoagulants are the most common cause of medication-related serious harm, in terms of emergency department visits, hospitalizations and fatalities. ${ }^{3,4}$ Transitions in care have been identified as a particularly high risk period for adverse events. ${ }^{5}$ Each adverse drug event requiring a hospital visit approximately doubles the cost of care in the subsequent 6 months. ${ }^{1}$ Indeed, our previous study of thromboembolic and hemorrhagic events after hospital discharge for patients taking oral anticoagulants (OACs), found rates to be approximately 2 to 3 times higher in the first month compared to later. ${ }^{6}$ Root cause analyses and patient safety inquiries cite problems with recognition of individual risk factors for benefit versus harm, drug interactions and contraindications, dosing adjustments over time and around procedures, drug monitoring and reversal strategies, and with communications and poor adherence by patients. ${ }^{7-9}$ Furthermore, the direct oral anticoagulants, each with different dosage regimens based on indication and no widely-available test toto measure the anticoagulant effect, have created confusion and increased opportunities for medication errors, while greatly increasing drug costs. ${ }^{2}$, 10,11

Anticoagulants are a high priority drug family for improved medication management because of a) established benefits to reduce the risk for stroke due to atrial fibrillation by approximately $70 \%$, the risk for recurrent venous thromboembolism by more than $90 \%$ and the risk of death by approximately $25 \%$, b) widespread utilization with more than $12 \%$ of people $>85$ years of age taking OACs and more than 7 million prescriptions dispensed annually in Canada, and c) ongoing under-prescribing of OACs for eligible patients, over-prescribing of reduced doses off-label, and use of inferior treatments such as aspirin. ${ }^{12-15}$

Although there are several well-developed anticoagulation management guidelines with detailed, evidence-informed recommendations, it is difficult for physicians and other health care providers to keep up with evolving evidence, and many patient situations lack high quality evidence to support a specific approach. ${ }^{16-18}$ Thrombosis Canada provides updated, user-friendly succinct clinical guidance for physicians and patients with point-of-care decision support tools, however the value of supplemental patient education and patient decision aids to improve outcomes remains unproven. ${ }^{19}$

Optimal medication safety requires not only best practices based on clinical evidence but also impeccable application and uptake. The latter requires coordination, communication with and education of all key providers, caregivers and the patient, as well as frequent monitoring, handovers and constant quality improvement. ${ }^{20-22}$ All of these may be most efficiently provided on a large scale across large 
regions by telehealth or virtual visits. ${ }^{23}$ However, the effectiveness and cost-effectiveness of telehealth interventions for medication management remain uncertain. ${ }^{24,25}$ In addition, methods of coordination and communication of care vary, with variable effect. ${ }^{26}$ Significantly, coordination interventions have rarely involved clinical pharmacologists - medical specialists with expertise in medication management and the ability to prescribe and change prescriptions.

We hypothesize that expert coordination and management of OAC therapy combined with frequent virtual visits by a pharmacist and clinical pharmacologist in the early post-hospital discharge period, plus regular communication with the patient's circle of care, could decrease adverse anticoagulant-related events and associated healthcare resource utilization while improving patient's health-related quality of life compared to usual care.

\section{Objectives}

The aim of this pilot randomized controlled trial (RCT) is to test whether high quality, easily scalable, expert multidisciplinary medication management and care coordination at hospital discharge and during short term post-discharge virtual visits, is feasible (primary) and can improve oral anticoagulant-related adverse event (thrombotic events, bleeds and deaths) rates, medication problems, quality-of-life, costeffectiveness, and satisfaction with care, (secondary) during a high-risk transition in care period.

\section{Methods And Analysis}

\section{Trial Design and Setting}

The protocol was developed according to the SPIRIT and TIDieR guidelines. ${ }^{27,28}$ This pragmatic pilot RCT is designed as a 2-arm, parallel, blinded assessment, variable block randomized trial with individual level randomization and outcomes of feasibility and clinical outcomes, during 3 months of follow-up.

Participating sites include 6 hospitals in Southwest Ontario - 3 academic teaching hospitals and 3 community hospitals. The trial was originally scheduled to begin in late 2019 but was delayed initially by staff shortages, then by Covid-19 restrictions on hospital-based research. ${ }^{29}$

\section{Eligibility Criteria}

Inclusion criteria include a) adult patients within a day of their hospital discharge from internal medicine services with a discharge prescription for an OAC intended to be taken for at least 4 weeks, b) discharge is to home or to a congregant setting such as retirement home where the patient manages their own medications, c) English-speaking and d) capable of providing informed consent. Ability to consent will be measured by the COACHeD Capacity to Consent test, requiring a score of 14 or more (Appendix 1). ${ }^{30}$ If the patient does not pass, a close caregiver (defined as a family member in daily contact with the 
patient and involved in their medication supervision), will be invited to provide consent on the patient's behalf by signing a caregiver consent form.

Patients will be excluded if they are less than 18 years of age, have an expected lifespan of less than 3 months, will be discharged to long term care or other institution where medications are controlled by staff, or decline informed consent.

\section{Intervention}

Figure 1 shows the study flow diagram including time and activities for both groups (see Additional File 1).

The Intervention Arm includes:

1. Interdisciplinary intervention led by a clinical pharmacologist who is a leader in evidence-based prescribing - includes a detailed discharge medication reconciliation and management plan focussed on oral anticoagulants at hospital discharge; a circle of care handover and coordination with patient, hospital team and community providers; three scheduled early post-discharge virtual medication check-up visits at 24 hours, 1 week, and 1 month with triage of any problems. Medication reconciliation is a process mandated by national accreditation bodies, with incomplete and variable uptake, which reviews hospital-administered medications compared to pre-admission medications. ${ }^{31}$ Medication management is the more complex task of assessing and revising medications in light of patient diagnoses, current symptoms and signs, risk factors, allergies and intolerances, other medications, goals, etc. In this study, all medications will be reviewd with a focus on OAC choice, dosage, indication, duration, potential drug interactions, patient risk factors for thromboembolism versus bleeding, drug insurance, adherence challenges and health literacy. A study pharmacist with additional training, will complete the detailed medication reconciliation.

2. Hand-overs to the community care team including the main patient caregiver (if applicable), family physician, medical specialist(s), and community pharmacist, using a templated consult summary including an OAC Monitoring Checklist. Figure 2 shows an example consult note (see Additional File 2). The monitoring is based on: a) best evidence (updated guidelines and dedicated evidence review using the CLOT repository of CanVECTOR and McMaster's Health Information Research Unit), and decision aid content for patients and their families to assist in anticoagulant knowledge and adherence, $b$ ) best practices regarding discharge medication management, virtual care, scalable coordination of care with clear accountability, communication and teletriage where situations require medical intervention. ${ }^{5,17,19,32-36}$. All consult notes are reviewed in detail with the Clinical Pharmacologist.

3. 'Virtual visits' (secure video calls from within our electronic medical record (EMR) or phone visits where video is not possible) by the study pharmacist at three follow-up time points - 24 hours postdischarge to ensure the discharge prescription medications were obtained and understood, OAC Monitoring Checklist, review other medications, solicit concerns; and at 1 week and 1 month to 
ensure medication adherence, review the OAC Monitoring Checklist and other medications, and solicit concerns. After each follow-up visit, a summary consult note will be sent to all circle of care providers, and any clinical events or serious concerns will be addressed by the Clinical Pharmacologist or directed to patient's family physician via phone call or direct email. Each followup visit with intervention patients will be recorded and tracked to ensure adherence to protocols.

4. Teletriage- The patients have the study pharmacist's contact information and can phone for assistance at any time. The study pharmacist is in constant communication with a Clinical Pharmacologist investigator for guidance. An expert Thrombosis specialist will be available on call as needed.

Control Arm: Patients allocated to the control group will receive usual care, plus the URL to Thrombosis Canada website. Usual care in the participating sites includes OAC management by family doctors except for new thromboembolic events which will be followed short term by thromboembolism or hematology specialists, complicated atrial fibrillation which will have cardiology involved temporarily, and a small proportion of warfarin management which is provided in an anticoagulation clinic. This choice of control group is the most relevant for generalizability to both academic and community practices.

For both arms, there are no restrictions placed on concomitant medical intervention or treatments, as this is a pragmatic randomized trial.

\section{Outcomes}

Study outcomes, their measurement methods, timing and analysis are presented in detail in Table 1 and Table 2, and include:

1. Primary outcomes, which are study feasibility outcomes, include recruitment and retention rates, and estimated resources required per patient to complete the main trial, We aim for at least 30\% recruitment of those eligible, $90 \%$ retention of those recruited, and no more than $\$ 1500$ per patient spent on running the trial. Secondary feasibility outcomes include barriers and facilitators to success of the primary outcomes, in terms of process and management issues. These will be used to determine whether a large definitive research study is likely to be feasible, taking into account the practical aspects of managing and funding the project. ${ }^{37,38}$

2. Secondary clinical outcomes include:

1. the Adverse Anticoagulant Safety Events composite (AASE) which is any of thromboembolic events or clinically relevant bleeding or death. Thromboembolic events include objectively verified ischemic stroke, systemic embolism, pulmonary embolism, or DVT. Clinically relevant bleeds in this study is defined as bleeding that causes death, hospitalization or Emergency Department visits.

2. Coordination and Continuity of Care: Adapted from Health Quality Ontario's draft guidance and a Rand instrument, $\{$ Health Quality Ontario, $2020 \# 5\}$ the Coordination and Continuity of Care 
Questionnaire is designed to measure the quality of the transitional and follow-up care. 5, 39,30

3. Patient Quality of Life: The EQ-5D-5L is the 5-level classification system of the EQ-5D, a measure of health status from the EuroQol Group..$^{40}$ Using EQ-5D-5L, respondents are asked a short series of questions about mobility, self-care, usual activities, pain discomfort, and anxiety/ depression, as well as a summary visual analogue scale. This scale, which provides utility measurements, has been well validated for the Canadian population. ${ }^{40-42}$

4. Patient Knowledge of OAC Management: Insufficient patient knowledge about OAC may predict poor medication adherence and inadequate anticoagulation control. ${ }^{43}$ The COACHeD OAC Knowledge Questionnaire tests knowledge of the therapeutic objective, process of use, safety and maintenance of the medications. ${ }^{30}$

5. Satisfaction with Care: Satisfaction reported by patients and by key health professionals is one of the recommended outcomes to report in medical research, as it may influence adherence. ${ }^{44,45}$ This outcome will be assessed by the Patient/Caregiver Study Satisfaction Survey and by the Provider Study Satisfaction Survey. ${ }^{30}$

6. Medication Problems: We will be assessing problems with appropriateness, with medication errors characterized using the NCC-MERP scale and with medication adherence and attitudes as measured by COMPETE Medication Problems Questionnaire. ${ }^{46,47}$ H Holbrook, 2011 \#80\} \{Holbrook, 2011 \#80\}

7. Resource Utilization: This is a key outcome to determine cost-effectiveness and cost-utility which then determines whether health care systems might pay for this type of care. ${ }^{48,49}$ We will be measuring all types of health care utilization by patients. ${ }^{39,41,49}$

\section{Sample Size Estimation}

Pilot RCT guidance suggest that sample size be based on the ability to detect a significant feasibility problem that might interfere with a subsequent full-size RCT - for example, difficulty with recruitment and retention, accuracy of outcome event capture and adjudication, frequency of events, and costing suggesting the intervention is not remotely cost-effective..$^{50}$ If a feasibility problem exists at a rate of $5 \%$ probability, it can be identified with $95 \%$ confidence with a sample of 59 participants. ${ }^{50}$

\section{Recruitment Methods}

Background work flow studies at several of the participating hospitals have confirmed that patient discharge is a complex, hurried process that is often rescheduled multiple times because of fluctuations in patient health or delays in required tests, procedures or milestones. For feasibility reasons, we plan to use a rolling recruitment method, spending two weeks at each hospital on a cyclical basis until a quota is recruited (10-12 patients per site). Rounds and recruitment posters will advertise the study, in some 
locations the EMR can assist with screening criteria, in all locations research staff are seeking eligible patients in consultation with the Most Responsible Physician team personnel.

\section{Allocation}

Participants who meet all the inclusion/exclusion criteria at screening and have completed informed consent will be enrolled in the study, complete baseline assessments, then will be randomized via a computer-generated randomization sequence stratified by site to one of the two study arms, intervention or control.

A statistician will prepare the randomization schedule using an adaptive biased-coin strategy. ${ }^{51}$ Randomization will be stratified by site to maintain balance and minimize the predictability of treatment assignments. ${ }^{52}$ To restrict treatment group imbalance a maximal tolerable imbalance between treatment groups will be incorporated into the schedule. ${ }^{53}$ The randomization schedule will be produced by a program written in SAS V9.4 software (SAS Institute Inc., Cary, NC, USA) and implemented in REDCap on a centralized computer where each patient's treatment assignment will be available on-line to the research pharmacist at the time of randomization. This process ensures allocation concealment, and randomization awareness where necessary, for example for the intervention staff.

\section{Blinding}

Since this is a pragmatic RCT of care coordination, it will not be possible to completely blind patients or their providers, however outcome data collectors, adjudicators and statisticians will be blinded to group allocation until analysis is completed at the end of the study.

\section{Data Collection Methods}

Trained research staff will conduct the interviews with the patients or caregivers, entering data electronically on study laptops directly into REDCap case report forms. The participants' medical records will be reviewed to abstract data on baseline characteristics, medical history, and medication information. Strategies to promote participant retention and complete follow-up include reminding participants in advance of their end-of-study visit and communicating by email if email address is provided at baseline. Participants who drop out of the study will have their data to that point retained in the study, as approved by REB, to avoid bias. The reasons for study non-completion will be recorded.

\section{Data Management}

REDCap (Research Electronic Data Capture) is our study software platform - secure, web-based, providing interfaces for validated data capture, role-specific access, audit trails for tracking data manipulation and exports, automated export procedures to SAS and encrypted transmissions. ${ }^{54,55}$ Paper study documents including signed informed consent forms will be stored in our secure research office once they are scanned into REDCap study files. Regular data quality checks, such as automatic range 
checks, will be performed by the study team to identify data that appear inconsistent, incomplete, or inaccurate.

Patients are not identifiable in the project results database. The identifying information required for the clinical team to deliver the intervention is kept in a separate database. Access to the final dataset will be restricted to the core research team.

\section{Statistical Analyses}

The reporting of the results of this trial will follow the CONSORT extension to pilot trials [ref]. We will use descriptive statistics for presentation of baseline variables and adequacy of follow-up. Feasibility analysis including recruitment rate ( $\geq 30 \%$ is considered success), participant retention rate ( $\geq 90 \%$ to end of study is considered success), study resource utilization required $(\$ 1,500$ per patient recruited and completing the study is considered a threshold), management assessment, and scientific assessment, will be descriptive.

Analysis will use intention-to-treat methods with censoring only if the patient dies or drops out of the study with refusal of negotiated further assessments. A sensitivity analysis of the subgroup of patients who received all 3 planned follow-up intervention calls and completed the end-study data collection (per protocol analysis), will be carried out. Research staff and statisticians will review outcome data and analysis blinded to group identification.

The primary clinical endpoint will be the incidence of adjudicated AASE during the follow-up period, using proportions, and chi-square testing. Secondary endpoint analyses of the coordination and continuity of care, patient quality of life, OAC management knowledge satisfaction with care (providers \& patients), resource utilization, will be analyzed using t-tests. The incidence of the adjudicated individual clinical events (clinically relevant bleeding events, thromboembolic events, all-cause hospitalizations and emergency department visits) will be analysed using the methods described above for AASE. Public unit costs from Ontario will be used to cost healthcare resource utilization collected as part of the trial. Using

an area under the curve approach, quality adjusted life years (QALYs) will be determined by weighting the EQ-5D-5L health utility scores by time spent in health state. Costs and outcomes (i.e., QALYs, AASE) between the interventions will be compared from a public payer perspective. Given the short follow-up, low risk of the trial and pilot design, no interim analysis or imputation for missing data is planned. All analyses will be performed using SAS V9.4 software (SAS Institute Inc., Cary, NC, USA).

\section{Data Monitoring}

Since this trial does not involve any investigational product or procedure, and uses the main anticoagulant safety events and medication safety as outcome events, a formal Data Safety Monitoring Board is not required. Any serious adverse event will be reviewed by our Trial Steering Committee (TSC) within a week of detection, to discern any attribution to our procedures. If found to be due to our 
coordination procedures, the trial steering committee will recommend whether modifications are indicated. The TSC will be composed of individuals with expertise in clinical trials, chaired by the lead statistician, include the PI, the operational statistician plus a methodologist independent of the study team. Similarly, since this is a short pilot pragmatic RCT where no harm is expected and adjustment of trial procedures may be necessary for feasibility, no formal external auditing of trial conduct is planned. There is no requirement for additional ancillary and post-trial care for those who might come to harm while in the trial, as usual medical care which covers this eventuality is already in place.

\section{Trial Management}

The overall Principal Investigator will be responsible for communicating any changes to the study, new information, or unanticipated events to the REB, to the sponsor, and to Local Principal Investigators (LPI). The LPI (also called Site Investigator) is responsible for supervising any individual or party to whom they have delegated tasks at the trial site. A Trial Management Group (TMG) will be responsible for the day-today management of the trial and will include at a minimum the overall PI, the local PIs and the trial Research Coordinator. The group will closely review all aspects of the conduct and progress of the trial, ensuring that there is a forum for identifying and addressing issues. Particular attention will be paid to progress towards trial milestones, adherence to the protocol and good research practices. Meetings will be minuted and retained in the study REDCap database.

\section{Patient Contributions}

Patients were specifically involved in the planning of this trial in several ways. A patient representative is a co-investigator. In preparation for this trial, we investigated barriers and facilitators to optimal OAC management, using a systematic review of the literature then a qualitative focus group study of the opinions of 26 patients/caregivers and 16 providers. ${ }^{56,57}$ These influenced the intervention content and timing. In addition, our choice of outcomes was guided by our recently published systematic survey of the literature regarding patient-important outcomes in OAC trials, as advised by patient groups. ${ }^{58}$ One of our Knowledge User - dissemination lead groups is ISMP Canada who are the national leads on medication safety with well developed 2-way communications with patients.

\section{Ethics AND DISSEMINATION}

The study has been approved (study \#1639) by Hamilton Integrated Research Ethics Board, Brant Community Healthcare System Research Ethics Committee, and by the Tri-Hospital Research Ethics Board of Waterloo. Significant protocol modifications will be proactively communicated to the research ethics boards through study amendments to obtain approval prior to the changes being implemented. Each modification will be assessed to determine whether it warrants communication with trial participants.

Dissemination will be through presentations, publications, our research social media, incorporation into our knowledge partner communications to stakeholders, and possible future grant application. The 
results of the trial will be reported first to trial collaborators. The main report will be drafted by

the trial coordinating team, and the final version will be agreed by the Trial Steering Committee

before submission for publication, on behalf of the collaboration. The trial will be reported in accordance with the Consolidated Standards of Reporting Trials (CONSORT) guidelines (www.consort-

statement.org).The results of the trial will be shared widely, and participants are able to request a copy of the results through contacting the local study team.

\section{Declarations}

Ethics Approval and Consent to Participate The study has been approved by the Hamilton, Waterloo and Brantford Research Ethics Boards. Dissemination will include publications, presentations, follow-up larger trials, and appropriate changes in national medication safety and thromboembolism guidelines driven by our investigators and knowledge use partners.

Prior to performing any trial-specific procedure, a signed consent form will be obtained for each participant. The consent form is shown in Appendix A. ${ }^{30}$ A delegated member of the trial team will discuss the study with the patient and relevant family members/caregivers and provide them with a study consent form which will describe the purpose of the trial, the procedures to be followed, and the risk/benefits of participation. Any questions that they might have will be answered by either the trial team or the investigator. Once this is completed, we will test the patient's capacity to consent (see questionnaire in Appendix 1) ${ }^{30}$ If the patient is found capable, they will be invited to sign the informed consent form. If not, a close caregiver who assists the patient with their medications and medical care, will be invited to sign. Consent will be voluntary and free from coercion. Both the person obtaining consent and the investigator will sign the informed consent form, and a copy will be given to the patient and caregiver (if applicable).

Consent for Publication Not applicable.

Availability of Data and Materials: An anonymized dataset will be shared in accordance with future requirements of our funders, the Canadian Institutes of Health Research.

Competing Interests None declared.

Funding This work was supported by Canadian Institutes of Health Research grant number FRN 148803 and the Hamilton Academic Health Services Organization grant number $\mathrm{HAH}-16-06$. The study funders had no role in the study design; collection, management, analysis, or interpretation of data; writing of the report; or the decision to submit the report for publication.

Authors' Contributions AH is the principal investigator and conceived the study idea, wrote the protocol, wrote the grant for funding, and leads the project team. SH, JD, SS, KK, JH, JET, DS, LT were involved in the design of the study and securing funding. $\mathrm{KA}$ is the patient representative. AH, ST, KV, LY drafted the 
protocol manuscript, applied for ethics approval, and coordinated the study. SG, BL, AA, MT, YK are local principal investigators. All authors have read and approved the final manuscript. AH acts as guarantor.

All authors conform to the Pilot and Feasibility Studies eligibility guidelines:

- Substantial contributions to the conception or design of the work; or the acquisition, analysis, or interpretation of data for the work; AND

- Drafting the work or revising it critically for important intellectual content; AND

- Final approval of the version to be published; AND

- Agreement to be accountable for all aspects of the work in ensuring that questions related to the accuracy or integrity of any part of the work are appropriately investigated and resolved.

No professional writers were used to write this manuscript.

Acknowledgements Not applicable.

\section{References}

1. IMSP. Hospital to Home - Facilitating Medication Safety at Transitions: A Toolkit for Healthcare Providers: ISMP; 2015 [Available from: www.ismp-canada.org.

2. Moore T CM, Furberg C, Mattison D. QuarterWatch 2013 Quarter 1: Perspective on Drug Hypersensitivity. ResearchGate. 2014:1-18.

3. Bayoumi I, Dolovich L, Hutchison B, Holbrook A. Medication-related emergency department visits and hospitalizations among older adults. Can Fam Physician. 2014;60(4):e217-22.

4. Budnitz DS, Shehab N, Lovegrove MC, Geller Al, Lind JN, Pollock DA. US Emergency Department Visits Attributed to Medication Harms, 2017-2019. JAMA. 2021;326(13):1299-309.

5. Ontario HQ. Transitions between Hospital and Home: Care for People of All Ages: HQO; 2020 [Available from: https://www.hqontario.ca/Portals/0/documents/evidence/quality-standards/qstransitions-between-hospital-and-home-quality-standard-en.pdf.

6. Holbrook A BH, Paterson M, Martins D, Greaves S, Munil P, Gomes T. Oral Anticoagulantassociated Adverse Event Rates are High in the Post-Hospital Discharge Period. CMAJ Open. 2021;9(2):364-75.

7. Graves CM, Haymart B, Kline-Rogers E, Barnes GD, Perry LK, Pluhatsch D, et al. Root Cause Analysis of Adverse Events in an Outpatient Anticoagulation Management Consortium. Jt Comm J Qual Patient Saf. 2017;43(6):299-307. 
8. Banerjee A, Benedetto V, Gichuru P, Burnell J, Antoniou S, Schilling RJ, et al. Adherence and persistence to direct oral anticoagulants in atrial fibrillation: a population-based study. Heart. 2020;106(2):119-26.

9. $\quad$ Yu A, Jeyakumar Y, Wang M, Lee J, Marcucci M, Holbrook A. How personalized are benefit and harm results of randomized trials? A systematic review. J Clin Epidemiol. 2020;126:17-25.

10. Majeed A, Schulman S. Bleeding and antidotes in new oral anticoagulants. Best Pract Res Clin Haematol. 2013;26(2):191-202.

11. Moore T CM, Furberg C. Monitoring FDA MedWatch Reports: Anticoagulants the Leading Reported Drug Risk in 2011. ISMP Quarterwatch; 2011.

12. Aguilar MI, Hart R. Oral anticoagulants for preventing stroke in patients with non-valvular atrial fibrillation and no previous history of stroke or transient ischemic attacks. Cochrane Database Syst Rev. 2005(3):CD001927.

13. Ben Freedman S, Gersh BJ, Lip GY. Misperceptions of aspirin efficacy and safety may perpetuate anticoagulant underutilization in atrial fibrillation. Eur Heart J. 2015;36(11):653-6.

14. Ogilvie IM, Newton N, Welner SA, Cowell W, Lip GY. Underuse of oral anticoagulants in atrial fibrillation: a systematic review. Am J Med. 2010;123(7):638-45 e4.

15. Sharma V, Koczka C, Fischer C. Underutilization of evidence-based strategies in the diagnosis and treatment of venous thromboembolism among trainees. J Hosp Med. 2010;5(1):E26-30.

16. Physicians ACoC. American College of Chest Physicians Evidence-Based Clinical Practice Guidelines: Antithrombotic Therapy and Prevention of Thrombosis; 2012 [Available from: http://journal.publications.chestnet.org/issue. aspx?issueid=23443.

17. Holbrook A, Schulman S, Witt DM, Vandvik PO, Fish J, Kovacs MJ, et al. Evidence-based management of anticoagulant therapy: Antithrombotic Therapy and Prevention of Thrombosis, 9th ed: American College of Chest Physicians Evidence-Based Clinical Practice Guidelines. Chest. 2012;141(2 Suppl):e152S-e84S.

18. Witt DM, Nieuwlaat R, Clark NP, Ansell J, Holbrook A, Skov J, et al. American Society of Hematology 2018 guidelines for management of venous thromboembolism: optimal management of anticoagulation therapy. Blood Adv. 2018;2(22):3257-91.

19. Douketis J BM, Bell A, et al. Clinical Guides 2014: Thrombosis Canada; 2014 [Available from: https://thrombosiscanada.ca/clinicalguides/.

20. De Regge M DPK, Meijboom B, Trybou J, Mortier E, Eekloo K. The role of hospitals in bridging the care continuum: A systemtic review of coordination of care and follow-up for adults with chronic 
conditions. BMC Health Serv Res. 2017(17).

21. Tricco AC AJ, Ivers NM, Ashoor HM, Khan PA, Blondal E, Ghassemi M, MacDonald H, Chen MH, Ezer LK, Straus SE. Effectiveness of quality improvement strategies for coordination of care to reduce use of health care services: A systematic review and meta-analysis. CMAJ Open. 2014;186(15):E568-E78.

22. Huntley A LD, Wye L, Morris R, Checkland K, England H, Salisbury C, Purdy S. Which feaures of primary care affect unscheduled secondary care use? A systematic review. BMJ Open. 2013;4(5).

23. Lee $M$, Wang M, Liu J, Holbrook A. Do telehealth interventions improve oral anticoagulation management? A systematic review and meta-analysis. J Thromb Thrombolysis. 2018;45(3):325-36.

24. Lee PA, Greenfield G, Pappas Y. The impact of telehealth remote patient monitoring on glycemic control in type 2 diabetes: a systematic review and meta-analysis of systematic reviews of randomised controlled trials. BMC Health Serv Res. 2018;18(1):495.

25. Ekeland AG, Bowes A, Flottorp S. Effectiveness of telemedicine: a systematic review of reviews. Int J Med Inform. 2010;79(11):736-71.

26. Duan-Porter W, Ullman K, Majeski B, Miake-Lye I, Diem S, Wilt TJ. Care Coordination Models and Tools: A Systematic Review and Key Informant Interviews. VA Evidence-based Synthesis Program Reports. Washington (DC)2020.

27. Chan AW, Tetzlaff JM, Altman DG, Dickersin K, Moher D. SPIRIT 2013: new guidance for content of clinical trial protocols. Lancet. 2013;381(9861):91-2.

28. Hoffmann TC, Glasziou PP, Boutron I, Milne R, Perera R, Moher D, et al. Better reporting of interventions: template for intervention description and replication (TIDieR) checklist and guide. BMJ. 2014;348:g1687.

29. Orkin AM, Gill PJ, Ghersi D, Campbell L, Sugarman J, Emsley R, et al. Guidelines for Reporting Trial Protocols and Completed Trials Modified Due to the COVID-19 Pandemic and Other Extenuating Circumstances: The CONSERVE 2021 Statement. JAMA. 2021;326(3):257-65.

30. COACHeD RCT Additional Files 2021 [Internet]. MacSphere, McMaster University. 2021. Available from: http://hdl.handle.net/11375/27213.

31. ISMPCanada. Medication Reconciliation (MedRec) ISMPCanada: ISMPCanada; 2000 [Available from: https://www.ismp-canada.org/medrec/.

32. ThrombosisCanada. Thrombosis Canada [internet] Canada: Thrombosis Canada; unknown [Available from: https://thrombosiscanada.ca/. 
33. Keshavjee K, Mirza K, Martin K. The Next Generation EMR. Stud Health Technol Inform. 2015;208:210-4.

34. ISMPCanada. Virutal Medication History Interviews and Discharge Education: ISMP; 2020 [Available from: www.ismp-canada.org.

35. Core Elements of Anticoagulation Stewardship Programs 2019: Anticoagulation Forum; 2019 [Available from:

https://acforum.org/web/downloads/ACF\%20Anticoagulation\%20Stewardship\%20Guide.pdf.

36. Unit MsHIR. CLOT Respository: McMaster University; [Available from: https://plus.mcmaster.ca/clotplus.

37. Eldridge SM, Chan CL, Campbell MJ, Bond CM, Hopewell S, Thabane L, et al. CONSORT 2010 statement: extension to randomised pilot and feasibility trials. Pilot Feasibility Stud. 2016;2:64.

38. Thabane L, Lancaster G. A guide to the reporting of protocols of pilot and feasibility trials. Pilot Feasibility Stud. 2019;5:37.

39. Wenger NS, Roth CP, Shekelle P, Investigators A. Introduction to the assessing care of vulnerable elders-3 quality indicator measurement set. J Am Geriatr Soc. 2007;55 Suppl 2:S247-52.

40. Euroqol. EQ-5D Instruments: Euroqol; 2018 [Available from: https://euroqol.org/eq-5dinstruments/.

41. Herdman M, Gudex C, Lloyd A, Janssen M, Kind P, Parkin D, et al. Development and preliminary testing of the new five-level version of EQ-5D (EQ-5D-5L). Qual Life Res. 2011;20(10):1727-36.

42. Xie F, Pullenayegum E, Gaebel K, Bansback N, Bryan S, Ohinmaa A, et al. A Time Trade-offderived Value Set of the EQ-5D-5L for Canada. Med Care. 2016;54(1):98-105.

43. Rolls CA OK, Chalmers $\mathrm{L}$, et al. The relationship between knowledge, health literacy, and adherence among patients taking oral anticoagulants for stroke thromboprophyxlaxis in atrial fibrillation. Cardiovasc Ther. 2017;35(6).

44. Williamson PR, Altman DG, Bagley H, Barnes KL, Blazeby JM, Brookes ST, et al. The COMET Handbook: version 1.0. Trials. 2017;18(Suppl 3):280.

45. Perino AC, Shrader P, Turakhia MP, Ansell JE, Gersh BJ, Fonarow GC, et al. Comparison of Patient-Reported Care Satisfaction, Quality of Warfarin Therapy, and Outcomes of Atrial Fibrillation: Findings From the ORBIT - AF Registry. J Am Heart Assoc. 2019;8(9):e011205.

46. Prevention NCCfMERa. About Medication Errors: MERP N; 2020 [Available from: https://www.nccmerp.org/about-medication-errors. 
47. Holbrook A, Pullenayegum E, Thabane L, Troyan S, Foster G, Keshavjee K, et al. Shared electronic vascular risk decision support in primary care: Computerization of Medical Practices for the Enhancement of Therapeutic Effectiveness (COMPETE III) randomized trial. Arch Intern Med. 2011;171(19):1736-44.

48. Carrasquillo O. Health Care Utilization. Encyclopedia of Behavioral Medicine. Gellman MD, Turner JR ed. New York: Springer; 2013. p. 909-10.

49. (CADTH) CAfDaTiH. Canadian Agency for Drug and Technogolies in Health [Internet] Canada: Canadian Journal of Health Technologies; 2021 [Available from: https://www.cadth.ca/.

50. Viechtbauer W, Smits L, Kotz D, Bude L, Spigt M, Serroyen J, et al. A simple formula for the calculation of sample size in pilot studies. J Clin Epidemiol. 2015;68(11):1375-9.

51. LJ W. The Adaptive Biased Coin Design for Sequential Experiments. The Annals of Statistics. 1978;6(1):92-100.

52. Markaaryan T RW. Exact properties of Efron's biased coin randomization procedure. Ann Statist. 2010;38(3):1546-67.

53. Soares JF JWC. Some restricted randomization rules in sequential designs. Communications in Statistics. 1983;12(17):2017-34.

54. Harris PA, Taylor R, Minor BL, Elliott V, Fernandez M, O'Neal L, et al. The REDCap consortium: Building an international community of software platform partners. J Biomed Inform. 2019;95:103208.

55. Harris PA, Taylor R, Thielke R, Payne J, Gonzalez N, Conde JG. Research electronic data capture (REDCap)-a metadata-driven methodology and workflow process for providing translational research informatics support. J Biomed Inform. 2009;42(2):377-81.

56. Holbrook A, Wang M, Swinton M, Troyan S, Ho JMW, Siegal DM. Barriers and facilitators for optimizing oral anticoagulant management: Perspectives of patients, caregivers, and providers. PLoS One. 2021;16(9):e0257798.

57. Wang M, Holbrook A, Lee M, Liu J, Leenus A, Chen N, et al. Barriers and facilitators to optimal oral anticoagulant management: a scoping review. J Thromb Thrombolysis. 2020;50(3):697-714.

58. Wang $M$, Chen $Z$, Wong M, Thabane L, Mbuagbaw L, Siegal D, et al. Are the correct outcomes being measured in studies of oral anticoagulants? A systematic survey. Thromb Res. 2021;201:30-49.

\section{Abbreviations}

COACHeD - Coordination of oral anticoagulant care at hospital discharge 
OAC - oral anticoagulant

RCT - randomized control trial

ISMP - Institute for Safe Medication Practices

AASE - adverse anticoagulant safety events

DVT - deep vein thrombosis

REDCap - Research Electronic Data Capture

QALY - qualify adjusted life years

EQ-5D-5L - European Quality of Life Five Dimension

CONSORT - consolidated standard of reporting trials

REB - research ethics board

$\mathrm{PI}$ - principal investigator

LPI - local principal investigator

TMG - trial management group

TSC - trial steering committee

EMR - electronic medical records

NCC-MERP -National Coordinating Council for Medication Error Reporting and Prevention

\section{Tables}

Table 1. Study Outcome Assessment 


\begin{tabular}{|c|c|c|c|c|c|}
\hline \multicolumn{2}{|l|}{ Outcomes* } & $\begin{array}{l}\text { Variable } \\
\text { type }\end{array}$ & $\begin{array}{l}\text { Criteria for } \\
\text { success }\end{array}$ & $\begin{array}{l}\text { Outcome } \\
\text { Measure }\end{array}$ & $\begin{array}{l}\text { Method of } \\
\text { Analysis }\end{array}$ \\
\hline \multicolumn{6}{|c|}{ A. Feasibility Outcomes } \\
\hline \multirow[t]{2}{*}{$\begin{array}{l}\text { Primary } \\
\text { feasibility } \\
\text { outcomes }\end{array}$} & $\begin{array}{l}\text { Participant } \\
\text { recruitment } \\
\text { rates }\end{array}$ & $\begin{array}{l}\text { Continuous } \\
\text { (\%) }\end{array}$ & $\begin{array}{l}\geq 30 \% \text { of those } \\
\text { eligible will be } \\
\text { considered } \\
\text { success }\end{array}$ & $\begin{array}{l}\text { Percentage } \\
\text { recruited and } \\
\text { rate of } \\
\text { recruitment (of } \\
\text { those screened, } \\
\text { of those eligible } \\
\text { and of those } \\
\text { approached) } \\
\text { Percentage of } \\
\text { those recruited } \\
\text { (signed } \\
\text { informed } \\
\text { consent) who } \\
\text { complete the } \\
\text { end of study } \\
\text { assessment at } 3 \\
\text { months }\end{array}$ & $\begin{array}{l}\text { Count of } \\
\text { percentages } \\
\text { and rates } \\
\text { over time }\end{array}$ \\
\hline & $\begin{array}{l}\text { Study Resource } \\
\text { Utilization }\end{array}$ & $\begin{array}{l}\text { Continuous } \\
\text { (\$CND) }\end{array}$ & $\begin{array}{l}<\$ 1500 \text { per } \\
\text { patient } \\
\text { recruited and } \\
\text { completing the } \\
\text { study }\end{array}$ & $\begin{array}{l}\text { Study costs per } \\
\text { patient } \\
\text { recruited }\end{array}$ & $\begin{array}{l}\text { Cost of } \\
\text { personnel, } \\
\text { supplies, } \\
\text { travel, etc } \\
\text { to run the } \\
\text { study/ \# } \\
\text { patients } \\
\text { recruited }\end{array}$ \\
\hline $\begin{array}{l}\text { Secondary } \\
\text { feasibility } \\
\text { outcomes }\end{array}$ & $\begin{array}{l}\text { Process } \\
\text { Assessments } \\
\text { Management } \\
\text { Assessments } \\
\text { Scientific } \\
\text { Assessments }\end{array}$ & $\begin{array}{l}\text { Mix of } \\
\text { continuous, } \\
\text { categorical } \\
\text { and } \\
\text { qualitative } \\
\text { data }\end{array}$ & $\begin{array}{l}\text { Implementation } \\
\text { is successful } \\
\text { and feasible, } \\
\text { questionnaires } \\
\text { are acceptable, } \\
\text { and data } \\
\text { gathered are } \\
\text { reliable and } \\
\text { valid }\end{array}$ & $\begin{array}{l}\text { Detailed } \\
\text { questions in } \\
\text { Feasibility } \\
\text { Assessment }\end{array}$ & Descriptive \\
\hline \multicolumn{3}{|c|}{ B. Clinical Outcomes } & & Hypothesis & \\
\hline $\begin{array}{l}\text { Primary } \\
\text { clinical } \\
\text { outcome }\end{array}$ & $\begin{array}{l}\text { Adverse } \\
\text { anticoagulant } \\
\text { safety events } \\
\text { composite } \\
\text { (AASE) }\end{array}$ & $\begin{array}{l}\text { Continuous } \\
\text { (\%) }\end{array}$ & $\begin{array}{l}\text { Intervention } \\
\text { group will have } \\
\text { fewer AASE } \\
\text { compared to } \\
\text { control group }\end{array}$ & $\begin{array}{l}\text { Count of AASE } \\
\text { at the end of } \\
\text { the study ( } 3 \\
\text { months). Rates } \\
\text { will be } \\
\text { expressed as } \\
\text { mean rate per } \\
\text { patient-year by } \\
\text { research } \\
\text { assistant. }\end{array}$ & $\begin{array}{l}\text { Chi-square } \\
\text { test }\end{array}$ \\
\hline $\begin{array}{l}\text { Secondary } \\
\text { clinical } \\
\text { outcomes }\end{array}$ & $\begin{array}{l}\text { Coordination and } \\
\text { continuity of } \\
\text { care }\end{array}$ & Continuous & $\begin{array}{l}\text { Intervention } \\
\text { will have } \\
\text { higher ratings } \\
\text { than control }\end{array}$ & $\begin{array}{l}\text { Coordination } \\
\text { and Continuity } \\
\text { of Care } \\
\text { Questionnaire } \\
\text { partially }\end{array}$ & T-tests \\
\hline
\end{tabular}


administered post-discharge and finalized at study end ( 3 months) by telephone.

\begin{tabular}{|c|c|c|c|c|}
\hline $\begin{array}{l}\text { Patient quality of } \\
\text { life }\end{array}$ & Continuous & $\begin{array}{l}\text { Intervention } \\
\text { will improve } \\
\text { more than } \\
\text { control }\end{array}$ & $\begin{array}{l}\text { EQ5D-5L - } \\
\text { difference in } \\
\text { mean change } \\
\text { scores at the } \\
\text { end of the } \\
\text { study. }\end{array}$ & T-tests \\
\hline $\begin{array}{l}\text { OAC Management } \\
\text { Knowledge - patient }\end{array}$ & Continuous & $\begin{array}{l}\text { Intervention } \\
\text { will have } \\
\text { higher scores } \\
\text { than control }\end{array}$ & $\begin{array}{l}\text { OAC Knowledge } \\
\text { Questionnaire }\end{array}$ & T-tests \\
\hline $\begin{array}{l}\text { Satisfaction with care } \\
\text { (providers \& patients) }\end{array}$ & Continuous & $\begin{array}{l}\text { Intervention } \\
\text { will have } \\
\text { higher scores } \\
\text { than control }\end{array}$ & $\begin{array}{l}\text { Satisfaction } \\
\text { Questionnaires }\end{array}$ & $\begin{array}{l}\text { Descriptive } \\
\text { analysis }\end{array}$ \\
\hline $\begin{array}{l}\text { Medication } \\
\text { Problems }\end{array}$ & Continuous & $\begin{array}{l}\text { Intervention } \\
\text { will have fewer } \\
\text { medication } \\
\text { problems than } \\
\text { control }\end{array}$ & $\begin{array}{l}\text { Composite of } \\
\text { OAC-APEQ, } \\
\text { COMPETE } \\
\text { adherence and } \\
\text { medication } \\
\text { error scores }\end{array}$ & T-tests \\
\hline $\begin{array}{l}\text { Cost- } \\
\text { effectiveness }\end{array}$ & Continuous & $\begin{array}{l}\text { Intervention } \\
\text { will be cost- } \\
\text { effective } \\
\text { compared to } \\
\text { control using a } \\
\text { threshold of } \\
\$ 50,000 \text { per } \\
\text { QALY. }\end{array}$ & $\begin{array}{l}\text { Cost per AASE } \\
\text { avoided and } \\
\text { cost per quality- } \\
\text { adjusted life- } \\
\text { years (QALYs) }\end{array}$ & $\begin{array}{l}\text { Economic } \\
\text { analysis }\end{array}$ \\
\hline $\begin{array}{l}\text { Individual } \\
\text { Clinical } \\
\text { Events: a) } \\
\text { Bleeding events } \\
\text { (clinically } \\
\text { relevant), b) } \\
\text { Thromboembolic } \\
\text { events (all); c) } \\
\text { Deaths; d) } \\
\text { Hospitalizations } \\
\text { or ED visits }\end{array}$ & Continuous & $\begin{array}{l}\text { Intervention } \\
\text { will have lower } \\
\text { event rates } \\
\text { than control }\end{array}$ & $\begin{array}{l}\text { Event rate } \\
\text { counts from } \\
\text { end-study } \\
\text { interviews }\end{array}$ & T-tests \\
\hline
\end{tabular}




\begin{tabular}{|c|c|c|c|c|c|}
\hline \multirow[b]{2}{*}{ ASSESSMENT } & \multicolumn{2}{|c|}{ Enrolment } & \multicolumn{2}{|c|}{ Study In-Progress } & \multirow{2}{*}{$\begin{array}{l}\text { Study } \\
\text { End } \\
3 \\
\text { months }\end{array}$} \\
\hline & Baseline & $\begin{array}{l}\text { Hospital } \\
\text { Discharge }\end{array}$ & $\begin{array}{l}24 \mathrm{hrs} \\
\quad 1 \\
\text { week }\end{array}$ & $\begin{array}{l}1 \\
\text { month }\end{array}$ & \\
\hline Eligibility Screen & $\mathrm{A}$ & & & & \\
\hline $\begin{array}{l}\text { COACHeD Brief Assessment of } \\
\text { Capacity to Consent } \\
\text { Informed Consent }\end{array}$ & $\begin{array}{l}\mathrm{B} \\
1 / \mathrm{C}\end{array}$ & & & & \\
\hline $\begin{array}{l}\text { Coordination Continuity of Care } \\
\text { Questionnaire (CCCQ) } \\
\text { EQ-5D-5L v10- Canada }\end{array}$ & $\begin{array}{l}\mathrm{I} / \mathrm{C} \\
1 / \mathrm{C}\end{array}$ & & & & $\mathrm{I} / \mathrm{C}$ \\
\hline OAC Knowledge Test & $\mathrm{I} / \mathrm{C}$ & & & & $\mathrm{I} / \mathrm{C}$ \\
\hline Randomization and Allocation & $\mathrm{I} / \mathrm{C}$ & & & & \\
\hline $\begin{array}{l}\text { Discharge Medication Reconciliation } \\
\text { and Management, } \\
\text { Circle of Care Communication and } \\
\text { Coordination } \\
\text { Virtual Visit Call \#1 }\end{array}$ & & I & I & & \\
\hline Virtual Visit Call \#2 & & & I & & \\
\hline Virtual Visit Call \#3 & & & & I & \\
\hline $\begin{array}{l}\text { Adverse Anticoagulant safety events } \\
\text { (AASE) }\end{array}$ & & & & & $\mathrm{I} / \mathrm{C}$ \\
\hline AASE Individual Components & & & & & $\mathrm{I} / \mathrm{C}$ \\
\hline $\begin{array}{ll}\text { Patient/Caregiver } & \text { Satisfaction } \\
\text { Questionnaire } & \end{array}$ & & & & & $\mathrm{I} / \mathrm{C}$ \\
\hline Provider Satisfaction Questionnaire & & & & & $\mathrm{I} / \mathrm{C}$ \\
\hline $\begin{array}{ll}\text { Health Resource } & \text { Utilization } \\
\text { Questionnaire } & \end{array}$ & & & & & $\mathrm{I} / \mathrm{C}$ \\
\hline Medication-related Problems & & & & & $\mathrm{I} / \mathrm{C}$ \\
\hline
\end{tabular}

\section{Figures}


Figure 1. COACHeD Flow Diagram

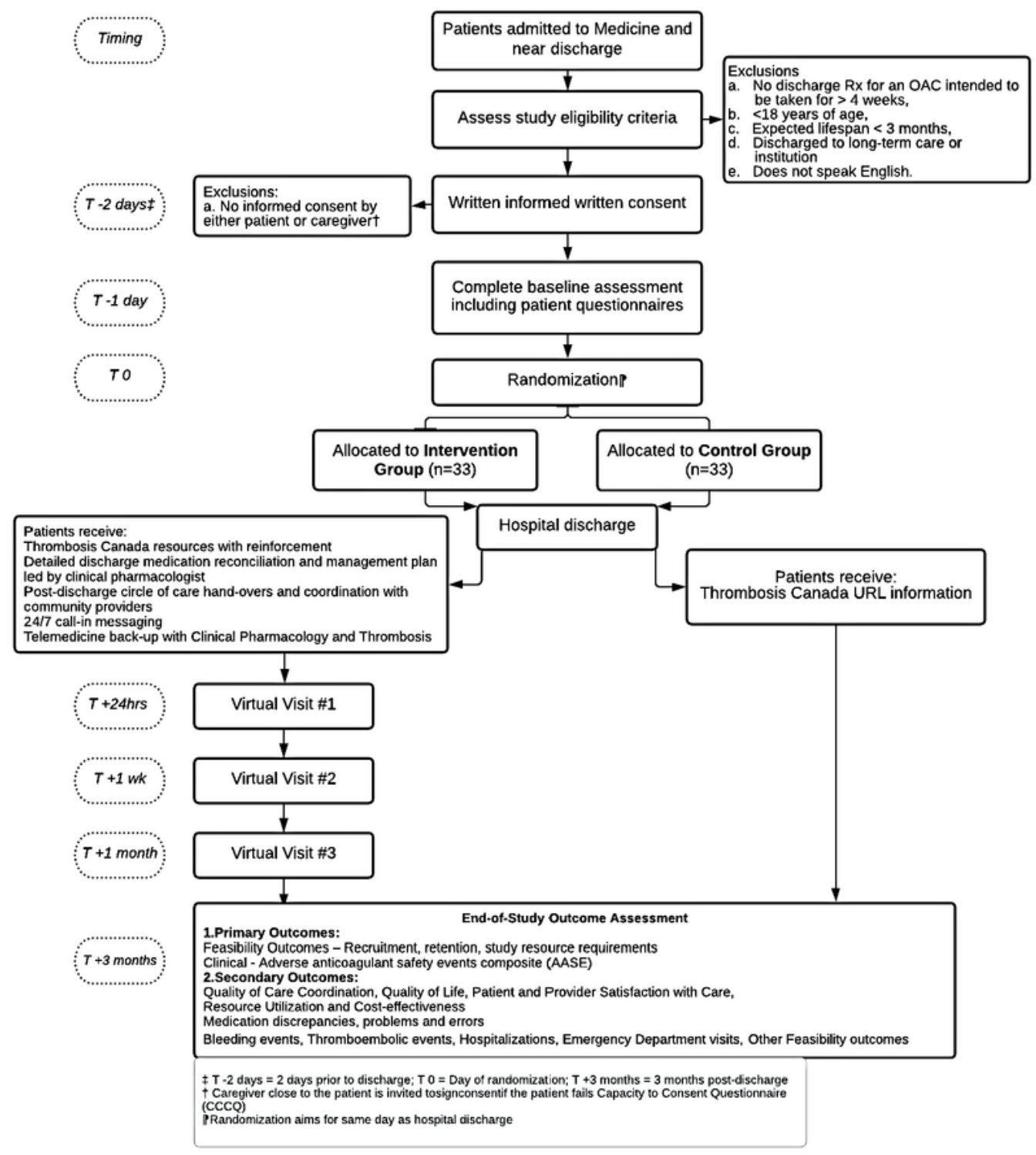

Figure 1

COACHeD Flow Diagram

Figures 2-4 are in the supplementary files section. 
Figure 2

Sample COACHeD Consult at Discharge

Figures 2-4 are in the supplementary files section.

\section{Figure 3}

SPIRIT Checklist

Figures 2-4 are in the supplementary files section.

\section{Figure 4}

TiDieR Checklist

\section{Supplementary Files}

This is a list of supplementary files associated with this preprint. Click to download.

- Appendix.docx

- Figure2COACHeDConsultLetterPage1.png

- Figure2COACHeDConsultLetterPage3.png

- Figure3SPIRITChecklistPage1.png

- Figure3SPIRITChecklistPage2.png

- Figure3SPIRITChecklistPage3.png

- Figure3SPIRITChecklistPage4.png

- Figure3SPIRITChecklistPage5.png

- Figure3SPIRITChecklistPage6.png

- Figure4TIDieRChecklistPage2.png

- Figure4TIDieRChecklistPage3.png 